\title{
Weight Loss and Gastrointestinal Symptoms in Advanced Cancer Patients Treated with Platinum-based Chemotherapy
}

\author{
Hui Gao ${ }^{1,2^{*}}$, Dong Bo Liu ${ }^{1 *}$, Jin Tong ${ }^{3}$, Jing Han ${ }^{1}$, Bo Liu ${ }^{1}$, Si Xian Zhu ${ }^{1}$, Liu Huang ${ }^{1}$, Ying Yao ${ }^{2}$, Shi \\ Ying $\mathrm{Yu}^{1}$, Qiang $\mathrm{Fu}^{1}$
}

\begin{abstract}
${ }^{1}$ Department of Oncology, Tongji Hospital, Tongji Medical College, Huazhong University of Science and Technology, Wuhan 430030, China; ${ }^{2}$ Department of Clinical Nutrition, Tongji Hospital, Tongji Medical College, Huazhong University of Science and Technology, Wuhan 430030, China; ${ }^{3}$ Department of Peripherally Inserted Central Venous Catheters (PICC), Tongji Hospital, Tongji Medical College, Huazhong University of Science and Technology, Wuhan 430030, China
\end{abstract}

\begin{abstract}
Objective This study assessed the weight loss changes and gastrointestinal symptoms in patients with advanced tumors receiving platinum-containing chemotherapy. Methods We retrospectively reviewed 297 patients with advanced cancers [124 gastrointestinal (GI) cancer patients, 119 lung cancer patients and 54 head and neck cancer (HNC) patients] receiving first-line chemotherapy at Tongji Hospital. The patients' changes in body weight, body mass index (BMI), and biochemical parameters (serum haemoglobin and albumin levels) were compared before and after two chemotherapy cycles. Results More than half [54.88\% (163/297)] of the patients had experienced unintentional weight loss in the 6 months before chemotherapy, and weight loss $\geq 5 \%$ and $\geq 10 \%$ of the body mass was noted in $35.69 \%$ and $20.20 \%$ of the patients, respectively. After two cycles of platinum-based chemotherapy, the proportions of patients with a $>5 \%$ reduction in body weight among patients with GI, lung, and head and neck cancers were 47.5\% (59/124), $44.53 \%$ (53/119), and 46.2\% (25/54), respectively. The patients with GI and lung cancers were more vulnerable to extreme weight loss $(\geq 10 \%)$ than those with HNC $(P=0.025)$. The serum hemoglobin levels were also remarkably decreased relative to those before chemotherapy (all $P<0.05$ ). Common GI symptoms reported by all patients included anorexia (61.28\%), vomiting (52.53\%), and nausea (51.18\%). A higher proportion of patients with $\geq 10 \%$ weight loss experienced anorexia and vomiting $(\mathrm{OR}=12.21$ and $3.61, P=0.008$ and 0.047 , respectively). Conclusions For advanced cancer patients receiving platinum-based chemotherapy, the GI symptoms are the major factor related to their nutritional status. Appropriate nutritional screening, evaluation and treatment should be applied during the treatment of cancer in order to reduce GI symptoms and improve the patient's nutritional status.
\end{abstract}

Key words: Weight loss; Platinum-based chemotherapy; Gastrointestinal symptoms; Hemoglobin

\section{Introduction}

Weight loss is common in cancer patients, and the incidence of unintentional weight loss ranges from $30 \% \sim 70 \%$ based on the type of malignant tumor [1]. It is regarded as a poor prognostic factor in patients with most types of cancer, both at initial disease presentation and during chemotherapy [2,3]. The extent of cancerinduced weight loss depends on the cancer type, stage and therapeutic modality used, and is multifactorial in etiology [4]. However, the contribution of potentially treatable symptoms to weight loss in patients at first presentation has been inadequately explored in the literature to date. A declining nutritional status and weight loss originate from multiple processes, but are associated with a decreased response to chemotherapy treatment and reduced survival [5]. Clinicians and patients must understand the effects of

\footnotetext{
"Hui Gao and Dong Bo Liu contributed equally to this manuscript. Corresponding author: Qiang Fu, MD, Department of Oncology, Tongji Hospital, Tongji Medical College, Huazhong University of Science and Technology, Wuhan 430030, China; Tel: +86 1896396 3732; Fax: +86 278366 2834; Email: fuqiang_tjh@126.com
}

malnutrition on patient outcomes, particularly in patients receiving chemotherapy.

Platinum-based chemotherapy (cisplatin, carboplatin, and oxaliplatin) is a treatment option common used for advanced gastrointestinal (GI), lung, and head \& neck cancers $[6,7]$. Nearly half of patients' experience grade 3-4 GI toxicities during platinum-based chemotherapy [8]. Thus, it is particularly important to pay attention to the nutritional status of patients with advanced cancer.

Nutritional deterioration has become an accepted part of the pathogenesis of cancer and its treatment. Nutritional screening includes anthropometric parameters [body mass index (BMI) and weight loss percentage] and biochemical parameters [hemoglobin ( $\mathrm{Hb})$ and albumin levels] $[9,10]$. There are often GI symptoms (nausea, vomiting, anorexia), weight loss, and decreases in the serum $\mathrm{Hb}$ and albumin levels in patients receiving chemotherapy [11]. A routine screen for malnutrition in cancer patients should include these factors.

The aim of this study was to observe the incidence of weight loss and assess the weight loss and nutritional status changes of advanced cancer patients receiving platinum- 
based chemotherapy.

\section{Materials and Methods Study patients}

Patients who received first-line chemotherapy for advanced cancer at the Cancer Center of Tongji Hospital from January 2018 to May 2019 were included in the analysis. We collected the patients' demographic and clinical characteristics from their electronic medical records. We retrospectively reviewed the records for patients who had received a definite diagnosis of cancer based on the evaluation of a pathological specimen and imaging examination. Eligibility criteria included an age between 18 and 75 years old; complete diagnostic information (primary tumor site, type of tumor and stage at diagnosis); complete information regarding the main GI symptoms that occurred during chemotherapy, which were reported by the patient's clinical oncology doctor (e.g. nausea, vomiting, diarrhea, constipation and anorexia, all recorded in the Chemotherapy Record Sheet in the electronic medical records); complete information for anthropometric variables (weight, height, usual weight, and weight loss during the 6 months before chemotherapy). Patients who received radiotherapy or who had a history of surgery as an oncological treatment were excluded from our study. A total of 1,104 patients were excluded from the analysis due to missing data. Finally, 297 patients were enrolled in this study (Figure 1). Biochemical and clinical data were assessed after the second chemotherapy cycle. For descriptive purposes, patients were classified by their tumor sites as follows: GI (gastric, colon, esophageal and gall bladder cancers); lung cancer; head and neck cancer (HNC).

The protocols involving human subjects/patients in the present study were approved by the Institutional Review Board of Tongji Hospital. All studies were conducted according to guidelines for biomedical research specified in the Declaration of Helsinki.

\section{Statistical analysis}

Descriptive statistics were used for the qualitative and quantitative variables, frequency, percentage, mean and standard deviation (SD). The average differences between groups were compared using Chi-squared tests (categorical variables) or a one-way ANOVA (continuous variables, normal distribution). After adjusting for age, a binary logistic regression model was used to examine the

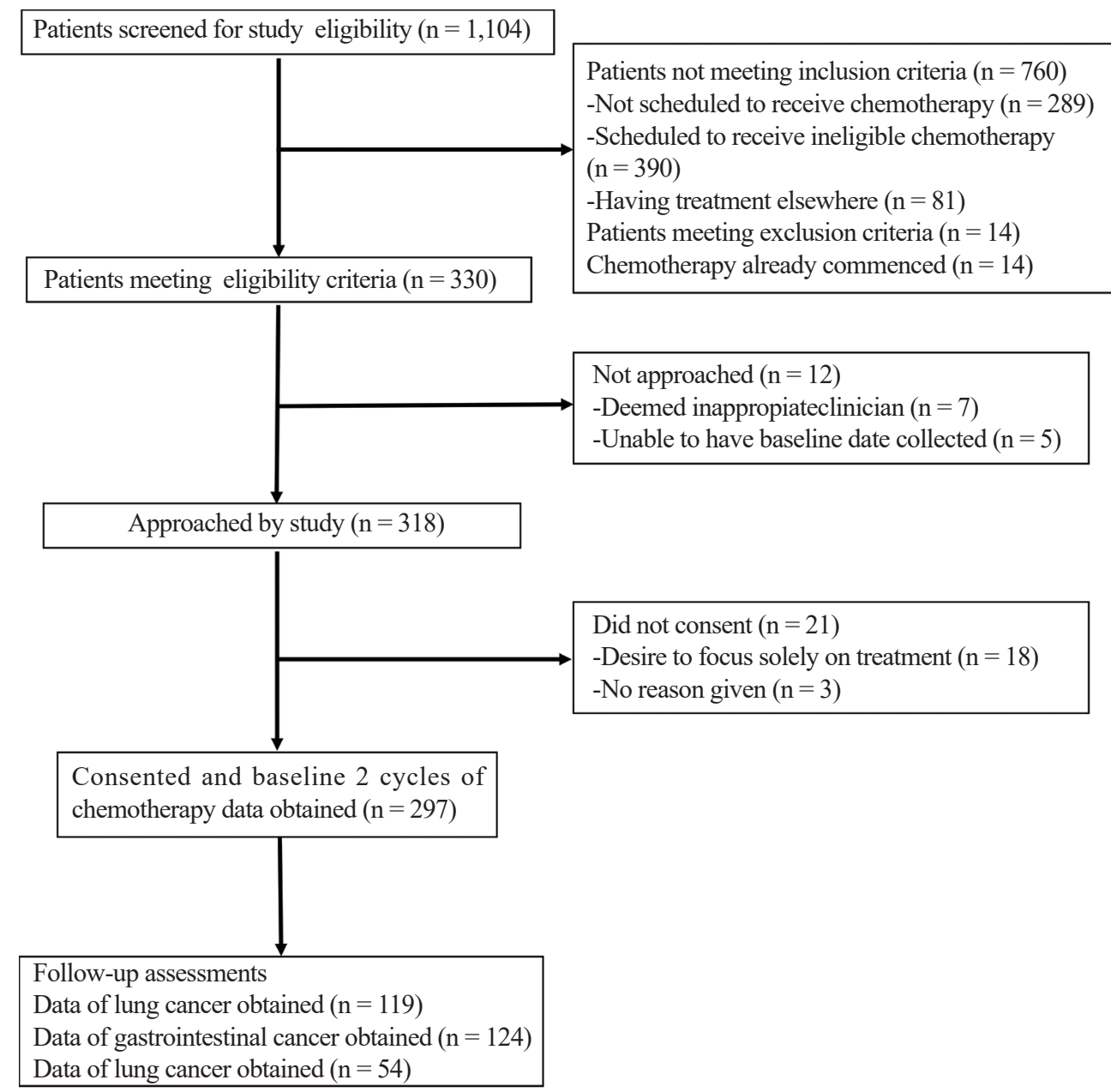

Figure 1 A CONSORT diagram reporting the numbers of individuals at each stage of the study. 
associations between weight loss and GI symptoms. Data analyses were performed using the SPSS program, version 16.0 (SPSS, Inc.). The $P$ values presented are two-tailed with a significance level of 0.05 .

\section{Results}

\section{Clinical characteristics}

A total of 297 cases met the inclusion criteria and were included in the analysis. Figure 1 summarizes the numbers of participants screened, approached and recruited. All patients received platinum chemotherapy, 189 (63.64\%) received a cisplatin or carboplatin regimen, and 108 $(36.36 \%)$ received oxaliplatin. The general characteristics of all enrolled patients are listed in Table 1. The patients were divided into GI cancer, lung cancer and HNC groups based on the location of the primary tumor, and these groups accounted for $41.75 \%, 40.07 \%$ and $18.18 \%$ of the patients, respectively. There were no significant differences among the three groups in terms of the gender distribution, initial weight or BMI (all $P>0.05$ ). Weight changes were common before chemotherapy, regardless of the type of tumors. During the 6 months before first-line chemotherapy was administered, $54.88 \%(163 / 297)$ of the patients experienced unintentional weight loss, and weight loss $\geq 5 \%$ and $\geq 10 \%$ of the body weight was reported by $35.69 \%$ and $20.20 \%$ of patients, respectively. It is worth noting that the age was significantly different among the three groups, with $\mathrm{HNC}$ patients being the youngest ( $48.31 \pm 12.61$ years).

\section{Changes of body weight and BMI before and after platinum-based chemotherapy}

After two cycles of platinum-based chemotherapy, $67.00 \%$ (199 of 297 patients) of the patients experienced unintentional weight loss (Table 2). The proportions of

Table 1 General characteristics of the patients.

\begin{tabular}{|c|c|c|c|c|c|}
\hline Tumor Location & Total & Gastrointestinal & Lung & Head and Neck & $P^{\mathrm{c}}$ \\
\hline $\mathrm{n}$ & 297 & $124(41.75 \%)$ & $119(40.07 \%)$ & $54(18.18 \%)$ & \\
\hline Gender $^{\mathbf{a}}$ & & & & & 0.226 \\
\hline Male & $188(63.30 \%)$ & $68(36.17 \%)$ & $82(43.62 \%)$ & $38(20.21 \%)$ & \\
\hline Female & $109(36.70 \%)$ & $56(51.38 \%)$ & $37(33.94 \%)$ & $16(14.68 \%)$ & \\
\hline Age (years) ${ }^{b}$ & $53.84 \pm 10.63$ & $54.48 \pm 11.98$ & $54.96 \pm 9.17$ & $48.31 \pm 12.61$ & $0.033^{*}$ \\
\hline Weight $(\mathrm{kg})^{\mathbf{b}}$ & $62.14 \pm 10.04$ & $62.50 \pm 11.46$ & $62.47 \pm 9.63$ & $59.93 \pm 10.78$ & 0.485 \\
\hline $\operatorname{BMI}\left(\mathrm{kg} / \mathrm{m}^{2}\right)^{\mathbf{b}}$ & $22.97 \pm 3.18$ & $23.08 \pm 3.91$ & $23.05 \pm 3.06$ & $22.40 \pm 3.03$ & 0.532 \\
\hline \multicolumn{6}{|c|}{$\begin{array}{l}\text { Weight loss during the } 6 \text { months } \\
\text { before chemotherapy }{ }^{\text {a }}\end{array}$} \\
\hline$\geq 5 \%$ body weight & $106(35.69 \%)$ & $45(42.45 \%)$ & $39(36.79 \%)$ & $22(20.75 \%)$ & 0.380 \\
\hline$\geq 10 \%$ body weight & $60(20.20 \%)$ & $27(45.00 \%)$ & $18(30.00 \%)$ & $15(25.00 \%)$ & 0.088 \\
\hline Chemotherapy regimen ${ }^{a}$ & & & & & - \\
\hline Cisplatin/Carboplatin & $189(63.64 \%)$ & $20(10.58 \%)$ & $119(62.96 \%)$ & $50(26.46 \%)$ & \\
\hline Oxaliplatin & $108(36.36 \%)$ & $104(96.30 \%)$ & $0(0)$ & $4(3.70 \%)$ & \\
\hline
\end{tabular}

-: No significance

${ }^{a}$ Data were presented as numbers (percentages) for categorical data.

${ }^{\mathbf{b}}$ Data were presented as means \pm SD for parametrically distributed data.

${ }^{\mathbf{c}}$ Differences between means were tested with a one-way ANOVA. Differences between medians were evaluated using a $\chi^{2}$ test.

* indicates a significant $(P<0.05)$ difference.

Table 2 Weight loss after 2 cycles of chemotherapy stratified by cancer location.

\begin{tabular}{lccccc}
\hline Type of cancer & $\mathbf{n}$ & \multicolumn{4}{c}{ Patients with weight loss [n (\%)] } \\
\cline { 3 - 6 } & & $\mathbf{< \%}$ body weight & $\mathbf{5 - 1 0 \%}$ body weight $\mathbf{2} \mathbf{1 0 \%}$ body weight & Total \\
\hline Gastrointestinal & 124 & $30(24.19)$ & $28(22.58)$ & $31(25.00)$ & $89(71.77)$ \\
Lung & 119 & $25(21.01)$ & $27(22.68)$ & $26(21.85)$ & $78(65.54)$ \\
Head and neck & 54 & $10(18.52)$ & $15(27.78)$ & $7(12.96)$ & $32(59.26)$ \\
Total & 297 & $65(21.89)$ & $70(23.57)$ & $64(21.55)$ & $199(67.00)$ \\
$P$ value & & 0.198 & 0.723 & 0.025 & 0.240 \\
\hline
\end{tabular}

Data were presented as numbers (percentages) for categorical data. 
participants who had a $5 \%$ or greater reduction in weight among participants with GI cancer, lung cancer, and HNC were 47.5\% (59/124), 44.53\% (53/119), 46.2\% (25/54), respectively. Patients who had GI $(25.00 \%, 31 / 124)$ and lung $(21.85 \%, 26 / 119)$ cancers were more vulnerable to extreme weight loss $(\geq 10 \%)$ than those with HNC $(18.52 \%, 10 / 54)(P=0.025)$. As shown in Table 3 , compared with the data recorded for patients before chemotherapy, the rate of weight loss was accelerated, especially in lung cancer patients $[(1.67 \pm 0.16) \mathrm{kg}$ vs. $(2.44 \pm 0.27) \mathrm{kg}, P=0.007]$. The magnitude of BMI reduction was nearly identical to that of weight reduction. Although there were no differences in patients with HNC in terms of the amount and rate of weight loss before chemotherapy and after 2-cycle chemotherapy (both $P$ $>0.05)$, the reduction of BMI increased gradually $(P=$ 0.007). To our surprise, the mean $\mathrm{Hb}$ levels in all patients were remarkably decreased relative to those before chemotherapy (all $P<0.05$ ). No significant difference was noted between the mean albumin levels before and after chemotherapy (all $P>0.05$ ).

\section{Gastrointestinal symptoms and weight loss}

Sixty-five percent of the patients were experiencing one or more GI symptoms at presentation. Symptoms were reported by $53 \%$ of men and $61 \%$ of women. Anorexia was the most common symptom $(61.28 \%)$, with vomiting $(52.53 \%)$ and nausea $(51.18 \%)$ being the next most common (Figure 2). Patients with GI and lung cancers exhibited similar patterns of symptoms. The patients with HNC were significantly more likely to report dysphagia than the patients with GI and lung tumors $(P=0.006)$. There were no significant differences between groups in terms of the number of patients reporting all other symptoms.

Table 3 Changes in body weight, BMI and biochemical parameters during chemotherapy.

\begin{tabular}{lccc}
\hline Variables & Before chemotherapy & After chemotherapy & $\boldsymbol{P}$ \\
\hline Weight $(\mathrm{kg})$ & $62.14 \pm 10.04$ & $61.29 \pm 10.33$ & $0.004^{* *}$ \\
Gastrointestinal & $62.50 \pm 11.46$ & $60.94 \pm 11.43$ & $0.045^{*}$ \\
Lung & $62.47 \pm 9.63$ & $61.68 \pm 10.02$ & $<0.01^{* *}$ \\
Head and Neck & $59.93 \pm 10.78$ & $59.59 \pm 11.00$ & 0.466 \\
Weight loss $(\mathrm{kg})$ & $1.78 \pm 0.16$ & $2.72 \pm 0.25$ & $0.001^{* *}$ \\
Gastrointestinal & $2.58 \pm 0.58$ & $4.24 \pm 0.82$ & 0.105 \\
Lung & $1.67 \pm 0.16$ & $2.44 \pm 0.27$ & $0.007^{* *}$ \\
Head and Neck & $1.43 \pm 0.34$ & $2.39 \pm 0.71$ & 0.174 \\
BMI $\left(\mathrm{kg} / \mathrm{m}^{2}\right)$ & $22.97 \pm 3.18$ & $22.58 \pm 3.31$ & $<0.01^{* *}$ \\
Gastrointestinal & $23.08 \pm 3.91$ & $22.46 \pm 3.26$ & $0.048^{*}$ \\
Lung & $23.05 \pm 3.06$ & $22.45 \pm 3.67$ & $0.001^{* *}$ \\
Head and Neck & $22.40 \pm 3.03$ & $22.15 \pm 3.25$ & 0.234 \\
BMI $\left(\mathrm{kg} / \mathrm{m}^{2}\right)$ & $0.35 \pm 0.09$ & $1.01 \pm 0.10$ & $<0.01^{* *}$ \\
Gastrointestinal & $0.61 \pm 0.28$ & $1.60 \pm 0.34$ & $<0.01^{* *}$ \\
Lung & $0.35 \pm 0.10$ & $0.89 \pm 0.10$ & $<0.01^{* *}$ \\
Head and Neck & $0.26 \pm 0.21$ & $0.91 \pm 0.27$ & $0.007^{* *}$ \\
Hemoglobin $(\mathrm{g} / \mathrm{L})$ & $130.30 \pm 19.23$ & $116.44 \pm 17.24$ & $<0.01^{* *}$ \\
Gastrointestinal & $115.86 \pm 23.32$ & $114.68 \pm 19.96$ & $<0.01^{* *}$ \\
Lung & $132.93 \pm 16.38$ & $116.08 \pm 16.17$ & $<0.01^{* *}$ \\
Head and Neck & $132.52 \pm 22.00$ & $120.36 \pm 19.76$ & $<0.01^{* *}$ \\
Albumin $(\mathrm{g} / \mathrm{L})$ & $37.93 \pm 4.46$ & $38.18 \pm 4.59$ & 0.488 \\
Gastrointestinal & $35.70 \pm 4.50$ & $37.22 \pm 4.72$ & 0.195 \\
Lung & $38.17 \pm 4.23$ & $38.13 \pm 4.49$ & 0.924 \\
Head and Neck & $39.13 \pm 4.42$ & $39.55 \pm 4.85$ & 0.618 \\
\hline
\end{tabular}

Data were presented as the means \pm SD for parametrically distributed data.

Differences between means were tested with paired-sample t-tests.

Before chemotherapy indicates the change during the 6 month period immediately before the patient received chemotherapy

After chemotherapy indicates the changes after 2 cycles of chemotherapy compared to baseline $\triangle$ BMI before chemotherapy = BMI 6 months before - BMI before chemotherapy. $\triangle$ BMI after chemotherapy $=$ BMI before chemotherapy - BMI after chemotherapy.

* indicates a significant $(P<0.05)$ difference.

** indicates a significant $(P<0.01)$ difference. 

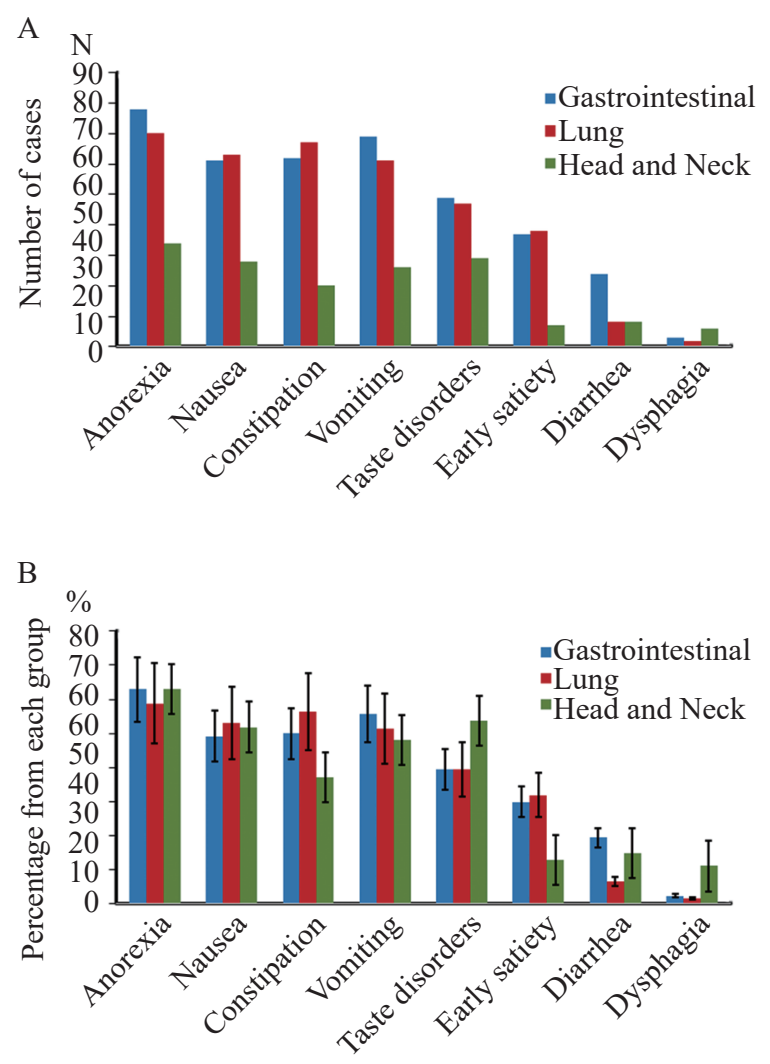

Figure 2 Gastrointestinal symptoms in the three groups of advanced cancer patients. The number (A) and percentage (with confidence intervals) (B) of patients with each symptom, according to the tumor type.

In the subset of patients $(n=199)$ with unintentional weight loss after chemotherapy, a planned analysis was undertaken to assess the relationship between GI symptoms and weight loss. Patients with $\geq 5 \%$ weight loss were significantly more likely to have experienced anorexia (OR $=9.02, P=0.011)$, nausea $(\mathrm{OR}=2.95, P=0.029)$, vomiting
$(\mathrm{OR}=2.79, P=0.012)$ and diarrhea $(\mathrm{OR}=3.14, P=$ $0.007)$. Weight loss $\geq 10 \%$ was significantly associated with anorexia $(\mathrm{OR}=12.21, P=0.008)$ and vomiting $(\mathrm{OR}=3.61$ $P=0.047$ ) (Table 4).

\section{Discussion}

The results of this study support the hypothesis that a patient's body weight and nutritional status are adversely affected by chemotherapy, and that chemotherapy-related effects are greatest early in the start of chemotherapy. To some extent, they are also associated with the primary tumor location, although these effects are variable, and patients with a variety of cancer types experience weight loss. The present study contributes new knowledge in the area of chemotherapy-related changes in body weight and the nutritional outcomes for patients receiving platinumbased chemotherapy for advanced cancer.

Of interest, we found that more than half of our patients had already experienced weight loss before chemotherapy. This may be explained by the tumor burden, due to factors such as the direct invasion of tumors into the GI tract or compression of digestive tissues, as well as to the hypermetabolism associated with tumors or the secretion of cytokines that cause cachexia[12,13]. In addition, the amount and rate of weight loss increased during cancer treatment. This acceleration of weight loss may be due to symptoms caused by the disease states, as well as the side effects of cancer chemotherapy, and the poor nutritional status will further reduce the patient's tolerance of the treatment $[14,15]$. Moreover, the fatigue, pain and psychological impact of the diagnosis and treatment can also reduce appetite[16-18].

The significant differences in the percentage weight loss and BMI among our patients serves as a reminder that the weight changes are also related to the primary tumor location $[19,20]$. BMI is an important nutritional index and

Table 4 Association between unintentional weight loss and gastrointestinal symptoms.

\begin{tabular}{|c|c|c|c|c|}
\hline \multirow{3}{*}{ Gastrointestinal symptoms } & \multicolumn{4}{|c|}{ Weight loss } \\
\hline & \multicolumn{2}{|c|}{$\geq 5 \%$ body weight } & \multicolumn{2}{|c|}{$\geq 10 \%$ body weight } \\
\hline & $\mathrm{OR}^{\mathrm{a}}(95 \% \mathrm{CI})$ & $P$ & $\mathrm{OR}^{\mathrm{a}}(95 \% \mathrm{CI})$ & $P$ \\
\hline Anorexia & $9.015(5.02,17.28)$ & 0.011 & $12.21(5.07,18.62)$ & 0.008 \\
\hline Nausea & $2.95(1.59,6.78)$ & 0.029 & $1.61(0.87,3.66)$ & 0.074 \\
\hline Constipation & $0.32(0.02,4.74)$ & 0.433 & $0.71(0.17,2.92)$ & 0.629 \\
\hline Vomiting & $2.79(1.03,4.32)$ & 0.012 & $2.68(0.65,5.09)$ & 0.033 \\
\hline Taste disorders & $1.410(0.69,2.89)$ & 0.349 & $1.29(0.33,4.97)$ & 0.072 \\
\hline Early satiety & $2.05(0.95,4.23)$ & 0.070 & $1.27(0.30,5.28)$ & 0.064 \\
\hline Diarrhea & $3.14(1.36,7.25)$ & 0.007 & $1.44(0.58,10.38)$ & 0.206 \\
\hline Dysphagia & $1.21(0.05,5.42)$ & 0.224 & $1.31(0.06,9.24)$ & 0.506 \\
\hline
\end{tabular}

Results from a binary logistic regression analysis are presented using the subset of patients $(n=199)$ with unintentional weight loss after chemotherapy.

OR, odds ratio; CI, confidence interval.

${ }^{\mathrm{a}}$ Adjusted for age. 
the simplest means to assess a patient's nutritional status. The mean decrease in BMI among our participants who underwent platinum-based chemotherapy was similar to that in a large-scale study involving 8,160 cancer patients. [4]. Of note, both underweight (BMI $<20 \mathrm{~kg} / \mathrm{m}^{2}$ ) and obese $\left(\mathrm{BMI}>30 \mathrm{~kg} / \mathrm{m}^{2}\right)$ patients represent groups for which therapy seems to be more challenging. Patients with a lower BMI were more likely to require reduced chemotherapy cycles and preterm discontinuation, while overweight or obese patients require higher medication intake and were more likely to develop chemotherapy-associated hematological toxicity [21]. In our study, the mean BMI was $(22.97 \pm 3.18) \mathrm{kg} / \mathrm{m}^{2}$; thus, the majority of our patients were considered to be at a normal weight. The patients with a lower BMI $\left(\mathrm{BMI}<20 \mathrm{~kg} / \mathrm{m}^{2}\right)$ did not show similar symptoms and trends as those with $\geq 5 \%$ weight loss.

It is still unclear why patients with cancer lose weight. The complexity of the disease etiology, as well as the potential overlapping presence of cachexia and malnutrition, demands caution when drawing any conclusions with regard to the cause and effect. In our study, we found that anorexia, vomiting and nausea were common in all of the patients, suggesting that platinum-based chemotherapy induces GI symptoms regardless of the primary tumor location. In contrast, the associations between weight loss and chemotherapy-related diarrhea and dysphagia were typically small or trivial. GI symptoms are important components of malnutrition in patients with cancer. In addition, changes in nutritional status have been associated with the altered absorption, metabolism and elimination of chemotherapy drugs.

The upper GI symptoms reported by patients are important because a high prevalence of these symptoms can cause difficulty in feeding, leading to a reduction of energy intake and worsening of the nutritional status $[22,23]$. Furthermore, the correlation between the number of symptoms and weight loss was weak but statistically significant, indicating that the GI symptoms may be responsible for weight loss. Therefore, during chemotherapy, promoting appetite and strengthening antiemetic therapy may be the most important factors to reduce weight loss and subsequently improve both the patient quality of life and the response to treatment [24]. All in all, the role of symptom management in improving nutritional status deserves further attention.

In line with other reports[25-27], the $\mathrm{Hb}$ level was significantly decreased after chemotherapy in our study, and was even out of the normal reference range (130$175 \mathrm{~g} / \mathrm{L})$. This result may be due to the toxic effects of the chemotherapeutic drugs on hematopoietic cells and the gut epithelium, which would lead to malabsorption [28].

Serum albumin is the simplest and most effective variable showing visceral protein function. It is commonly used in assessing malnutrition. In addition, the serum albumin concentration has been established as an independent prognostic variable for survival in patients with advanced GI cancer, lung cancer and HNC [2931]. Nevertheless, scarce data are available regarding the prevalence and clinical significance of hypoalbuminemia in patients with cancer and the manner by which this condition affects cancer treatment. Of note, the present results showed that the albumin levels were all normal, both before and after chemotherapy. The discrepancy between these results and previous studies may be due to the small sample size and short investigation time in the current work.

It should be acknowledged that our study is associated with several limitations. First, this study collected and analyzed data retrospectively, and some toxicity symptoms may have been under-reported. Second, oxaliplatin was used more frequently for gastrointestinal tumors than the other two platinum agents in this study, which may have impacted the results. This may also represent selection bias. Finally, weight loss per se may not accurately reflect the nutritional status in cancer patients, because it may be influenced by metabolic abnormalities, which result in a disproportionate loss of muscle mass. Thus, a body composition analysis should be introduced in subsequent research. In addition, we did not perform nutritional risk screening and assessment at baseline, nor dietetic screening. A relatively large sample size enabled us to achieve sufficient statistical power for the current analysis, but there may have been some unintentional bias due to the exclusion of patients with missing data. The nutritional interventions in this study included dietary advice, oral supplementation or major interventions (enteral feeding/tube placement), and the effects of different types of interventions should be taken into account.

\section{Conclusion}

In this study, HNC, GI and lung cancer patients all had an apparently higher prevalence of weight loss after platinum-based chemotherapy. GI symptoms such as anorexia and vomiting were significantly correlated with weight loss. The weight loss history, GI symptoms and $\mathrm{Hb}$ are all associated with the nutritional status in patients with advanced cancer. These factors must be included in the screening, evaluation and treatment of cancer patients, and appropriate nutritional support is important for maintaining the treatment intensity and may influence the outcomes of treatment.
Abbreviations
BMI, Body mass index;
$\mathrm{Hb}$, Hemoglobin;
SD, Standard deviation;
GI , Gastrointestinal;
$\mathrm{HNC}$, Head and neck cancer

\section{Acknowledgments}

The authors would like to thank all of the participants 
for their involvement in the study.

\section{Conflict of Interests}

No conflict of interest was declared by the authors.

\section{Contributors}

Qiang Fu and Hui Gao designed the project, performed data analysis, and wrote the paper. Dong Bo Liu carried out data processing, performed data analysis, and contributed to the paper writing. Jing Han, Jin Tong, Bo Liu, Si Xian Zhu and Liu Huang contributed to data processing. Ying Yao and Shi Ying Yu revised the paper.

\section{Funding}

This work was supported by the National Natural Science Foundation of China (No. 81703215, 81974381) and Beijing Xisike Clinical Oncology Research Foundation (No.Y-Q201801-059, 81974381).

\section{References}

1. Dewys WD, Begg C, Lavin PT, Band PR, Bennett JM, Bertino JR, Cohen MH, Douglass HO, Jr., Engstrom PF, Ezdinli EZ, Horton J, Johnson GJ, Moertel CG, Oken MM, Perlia C, Rosenbaum C, Silverstein MN, Skeel RT, Sponzo RW, Tormey DC. Prognostic effect of weight loss prior to chemotherapy in cancer patients. Eastern Cooperative Oncology Group. Am J Med 1980; 69(4):491-7.

2. Ryan AM, Power DG, Daly L, Cushen SJ, Ni Bhuachalla E, Prado CM. Cancer-associated malnutrition, cachexia and sarcopenia: the skeleton in the hospital closet 40 years later. Proc Nutr Soc 2016;75(2):199211.

3. Bozzetti F, Group SW. Screening the nutritional status in oncology: a preliminary report on 1,000 outpatients. Support Care Cancer 2009; 17(3):279-84.

4. Martin L, Senesse P, Gioulbasanis I, Antoun S, Bozzetti F, Deans C, Strasser F, Thoresen L, Jagoe RT, Chasen M, Lundholm K, Bosaeus I, Fearon KH, Baracos VE. Diagnostic criteria for the classification of cancer-associated weight loss. J Clin Oncol 2015;33(1):90-9.

5. von Haehling S, Anker SD. Cachexia as a major underestimated and unmet medical need: facts and numbers. J Cachexia Sarcopenia Muscle 2010;1(1):1-5.

6. Ndagi U, Mhlongo N, Soliman ME. Metal complexes in cancer therapy - an update from drug design perspective. Drug Des Devel Ther 2017;11:599-616.

7. Matthew T, Alex F. Health impact analysis of cisplatin, carboplatin and oxaliplatin. Johnson Matthey Technol Rev 2017;61(1):32-9.

8. Shahid F, Farooqui Z, Khan F. Cisplatin-induced gastrointestinal toxicity: an update on possible mechanisms and on available gastroprotective strategies. Eur J Pharmacol 2018;827:49-57.

9. Srdic D, Plestina S, Sverko-Peternac A, Nikolac N, Simundic AM, Samarzija M. Cancer cachexia, sarcopenia and biochemical markers in patients with advanced non-small cell lung cancer-chemotherapy toxicity and prognostic value. Support Care Cancer 2016;24(11):4495-502.

10. Lipkin EW, Bell S. Assessment of nutritional status. The clinician's perspective. Clin Lab Med 1993;13(2):329-52.

11. Tisdale MJ. Are tumoral factors responsible for host tissue wasting in cancer cachexia? Future Oncol 2010;6(4):503-13.

12. Costa RGF, Caro PL, de Matos-Neto EM, Lima J, Radloff K, Alves MJ, Camargo RG, Pessoa AFM, Simoes E, Gama P, Cara DC, da Silva ASF, W OP, Maximiano LF, de Alcantara PSM, Otoch JP, Trinchieri G, Laviano A, Muscaritoli M, Seelaender M. Cancer cachexia induces morphological and inflammatory changes in the intestinal mucosa. J Cachexia Sarcopenia Muscle 2019;10(5):1116-27.

13. Damrauer JS, Stadler ME, Acharyya S, Baldwin AS, Couch ME, Guttridge DC. Chemotherapy-induced muscle wasting: association with NF-kappaB and cancer cachexia. Eur J Transl Myol 2018;28(2):7590.

14. Eu CW, Ajit Singh V, Yasin NF. Effective nutritional status screening in orthopaedic oncology patients and post-operative complications. J Orthop Surg (Hong Kong) 2019;27(2):2309499019847232.

15. Haverkort EB, Binnekade JM, Busch OR, van Berge Henegouwen MI, de Haan RJ, Gouma DJ. Presence and persistence of nutrition-related symptoms during the first year following esophagectomy with gastric tube reconstruction in clinically disease-free patients. World $\mathrm{J}$ Surg 2010;34(12):2844-52.

16. Bye A, Jordhoy MS, Skjegstad G, Ledsaak O, Iversen PO, Hjermstad MJ. Symptoms in advanced pancreatic cancer are of importance for energy intake. Support Care Cancer 2013;21(1):219-27.

17. Blum D, Omlin A, Baracos VE, Solheim TS, Tan BH, Stone P, Kaasa S, Fearon K, Strasser F, European Palliative Care Research C. Cancer cachexia: a systematic literature review of items and domains associated with involuntary weight loss in cancer. Crit Rev Oncol Hematol 2011;80(1):114-44.

18. Sanchez-Lara K, Ugalde-Morales E, Motola-Kuba D, Green D. Gastrointestinal symptoms and weight loss in cancer patients receiving chemotherapy. Br J Nutr 2013;109(5):894-7.

19. Smith JL, Malinauskas BM, Garner KJ, Barber-Heidal K. Factors contributing to weight loss, nutrition-related concerns and advice received by adults undergoing cancer treatment. Adv Med Sci 2008;53(2):198-204.

20. Argiles JM, Busquets S, Stemmler B, Lopez-Soriano FJ. Cancer cachexia: understanding the molecular basis. Nat Rev Cancer 2014;14(11):754-62.

21. Grabowski JP, Richter R, Rittmeister H, Chekerov R, Woopen H, Sehouli J. Impact of Body Mass Index (BMI) on chemotherapy-associated toxicity in ovarian cancer patients. A pooled analysis of the North-Eastern German Society of Gynecological Oncology (NOGGO) databank on 1,213 Patients. Anticancer Res 2018;38(10):5853-8.

22. Souza NC, Simões BP, Júnior AA. Changes in intestinal permeability and nutritional status after cytotoxic therapy in patients with cancer. Nutrition and cancer, 2014,66(4):576-82.

23. Davidson W, Teleni L, Muller J, Ferguson M, McCarthy AL, Vick J, Isenring E. Malnutrition and chemotherapy-induced nausea and vomiting: implications for practice. Oncol Nurs Forum 2012;39(4):E340-5.

24. Buccheri G, Ferrigno D. Importance of weight loss definition in the prognostic evaluation of non-small-cell lung cancer. Lung Cancer 2001;34(3):433-440

25. Vadhan-Raj S, Dahl NV, Bernard K, Li Z, Strauss WE. Efficacy and safety of IV ferumoxytol for iron deficiency anemia in patients with cancer. J Blood Med 2017;8:199-209.

26. Topkan E, Selek U, Ozdemir Y, Yildirim BA, Guler OC, Mertsoylu H, Hahn SM. Chemoradiotherapy-induced hemoglobin nadir values 
and survival in patients with stage III non-small cell lung cancer. Lung Cancer 2018;121:30-6.

27. Jomrich G, Hollenstein M, John M, Ristl R, Paireder M, Kristo I, Asari R, Schoppmann SF. High mean corpuscular volume predicts poor outcome for patients with gastroesophageal adenocarcinoma. Ann Surg Oncol 2019;26(4):976-85.

28. Alexandre J, Gross-Goupil M, Falissard B, Nguyen ML, Gornet JM, Misset JL, Goldwasser F. Evaluation of the nutritional and inflammatory status in cancer patients for the risk assessment of severe haematological toxicity following chemotherapy. Ann Oncol 2003;14(1):36-41. 29. Arrieta O, Michel Ortega RM, Villanueva-Rodriguez G, Serna-Thome MG, Flores-Estrada D, Diaz-Romero C, Rodriguez CM,
Martinez L, Sanchez-Lara K. Association of nutritional status and serum albumin levels with development of toxicity in patients with advanced non-small cell lung cancer treated with paclitaxel-cisplatin chemotherapy: a prospective study. BMC Cancer 2010;10:50.

30. Pan YP, Hsu TY, Lin JY, Ho CJ, Kuan CY, Chou WC, Lai CH, Chang PH, Yeh KY. Prognostic significance of low body mass index and betel-quid use in the 5-year survival rates of esophageal squamous cell carcinoma patients. Nutr Cancer 2018;70(8):1315-21.

31. Danan D, Shonka DC Jr, Selman Y, Chow Z, Smolkin ME, Jameson MJ. Prognostic value of albumin in patients with head and neck cancer. Laryngoscope 2016;126(7):1567-71. 